\title{
Parabolic Equations with a Singular Absorption: Extinction Versus Blow-UP
}

\author{
Nguyen Anh DAO \\ Applied Analysis Research Group, Faculty of Mathematics and Statistics, \\ Ton Duc Thang University, Ho Chi Minh City, Vietnam \\ daonguyenanh@tdt.edu.vn \\ (Received: 12-September-2017; accepted: 25-September-2017; published: 30-November-2017) \\ DOI: http://dx.doi.org/10.25073/jaec.201712.63
}

\begin{abstract}
We prove a local existence of weak solutions of semilinear parabolic equations with a strong singular absorption and a source. Moreover, we consider the qualitative behavior of solutions. We show that any solution exists globally and vanishes after a finite time if either the initial data or the source term is small enough. On the other hand, we point out some criteria such that solutions are explosive in a finite time.
\end{abstract}

\section{Keywords}

Nonlinear parabolic equations, quenching phenomenon, singular absorption.

\section{Introduction}

In this paper, we are interested in nonnegative solutions of the following equation:

$\left\{\begin{array}{lr}\partial_{t} u-\Delta u+u^{-\beta} \chi_{\{u>0\}}=f(u) & \text { in } \Omega \times(0, T), \\ u(x, t)=0 & \text { on } \partial \Omega \times(0, T), \\ u(x, 0)=u_{0}(x) & \text { in } \Omega,\end{array}\right.$

where $\Omega$ is a bounded domain in $\mathbb{R}^{N}, 0<\beta<1$, and $\chi_{\{u>0\}}$ denotes the characteristic function of the set of points $(x, t)$ where $u(x, t)>0$, i.e:

$$
\chi_{\{u>0\}}= \begin{cases}1, & \text { if } u>0 \\ 0, & \text { if } u \leq 0\end{cases}
$$

Note that the absorption term $u^{-\beta} \chi_{\{u>0\}}$ becomes singular when $u$ is near to 0 , and we impose tactically $u^{-\beta} \chi_{\{u>0\}}=0$ whenever $u=0$.

Problem Eq. (1) can be considered as a limit of mathematical models describing enzymatic kinetics (see 11), or the Langmuir-Hinshelwood model of the heterogeneous chemical catalyst (see, e.g. 19] p. 68, 22, [18). This problem has been studied by the authors in [18, 29, [28, [13, [16], [9, 17, [8, 6], 20], and references therein. These authors have considered the existence and uniqueness, and the qualitative behavior of these solutions. For example, when $f=0$, D. Phillips 18 proved the existence of solution for the Cauchy problem associating to Eq. (1). A partial uniqueness of solution of Eq. (1) was proved by J. Davila and M. Montenegro, [9] for a class of solutions with initial data $u_{0}(x) \geq C \operatorname{dist}(x, \partial \Omega)^{\mu}$, for $\mu \in\left(1, \frac{2}{1+\beta}\right)$ (see also [5] the uniqueness in a different class of solutions). A beautiful result established by M. Winkler, 20], showed that the uniqueness of solution fails in general. One of the interesting behaviors of solutions of Eq. (1) is the extinction that any solution vanishes after a finite time even beginning with a positive initial data, see [18, [28] ( see also [7] for a quasilinear equation of this type). It is known that this phenomenon occurs according to the presence of the nonlinear singular absorption $u^{-\beta} \chi_{\{u>0\}}$. One can see the same situation for the nonlinear absorption $u^{\beta}$, for $\beta \in(0,1)$, see 21 and references therein. 
Equation (1) with source term $f(u)$ satisfying the sublinear condition, i.e: $f(u) \leq C(u+1)$, was considered by J. Davila and M. Montenegro, [9]. The authors proved the existence of solution and showed that the measure of the set $\{(x, t) \in \Omega \times(0, \infty): u(x, t)=0\}$ is positive (see also a more general statement in [23]). In other words, the solution may exhibit the quenching behavior. Still in the sublinear case with source term $\lambda f(u)$, M. Montenegro [17, proved that there is a real number $\lambda_{0}>0$ such that for any $\lambda \in\left(0, \lambda_{0}\right)$, there is $t_{0}>0$ such that

$$
u\left(x, t_{0}\right)=0, \quad \forall x \in \Omega .
$$

And it is called the complete quenching phenomenon.

From our knowledge, Eq. (1) with a general source term $f(u)$ has not been studied completely, such as $f(u)=u^{2}$. Thus, we would like to investigate existence, and the qualitative behavior of solutions of Eq. (1) for source term $f(u)$. It is well known that nonlinear parabolic equations with general source $f(u)$ may cause the finite time blow-up, i.e: there is a time $T_{0}>0$ such that $\lim _{t \rightarrow T_{0}}\|u(t)\|_{\infty} \rightarrow+\infty$. As mentioned above, the nonlinear absorption $u^{-\beta} \chi_{\{u>0\}}$ causes the complete quenching phenomenon. Thus, it is interesting to see when the complete quenching prevails the blow-up, and conversely. We also note that the similar questions were studied by [7, [8], [6] for the quasilinear parabolic equations of this type. To be simple, we consider $f(u)=u^{p}, p \geq 1$ through this paper, although our analysis can be applied to a general source $f(u)$, which is a locally Lipschitz function on $[0, \infty)$. Before discussing the behaviors of solutions of Eq. (1), it is necessary to introduce a notion of weak solution, and establish first a local existence of solutions of Eq. (1).

Definition 1. Let $u_{0} \in L^{\infty}(\Omega)$. A nonnegative function $u(x, t)$ is called a weak solution of Eq. I1 if $u^{-\beta} \chi_{\{u>0\}} \in L^{1}(\Omega \times(0, T))$, and $u \in L^{p}\left(0, T ; W_{0}^{1,2}(\Omega)\right) \cap L^{\infty}(\Omega \times(0, T)) \cap$ $\mathcal{C}\left([0, T) ; L^{1}(\Omega)\right)$ satisfies Eq. (1) in the sense of distributions $\mathcal{D}^{\prime}(\Omega \times(0, T))$, i.e:

$$
\int_{0}^{T} \int_{\Omega}\left(-u \phi_{t}+\nabla u . \nabla \phi+u^{-\beta} \chi_{\{u>0\}} \phi-f(u) \phi\right) d x d t=0
$$

for all $\phi \in \mathcal{C}_{c}^{\infty}(\Omega \times(0, T))$.
Our local existence result is as follows.

Theorem 1. Let $u_{0} \in L^{\infty}(\Omega)$, and $f(u)=u^{p}$. Then, there exists a finite time $T=T\left(u_{0}\right)>0$ such that Eq. (1) has a maximal weak solution $u$ in $\Omega \times(0, T)$, i.e: for any weak solution $v$ in $\Omega \times(0, T)$, we have

$$
v \leq u, \quad \text { in } \Omega \times(0, T) .
$$

Moreover, there is a positive constant $C=$ $C\left(\left\|u_{0}\right\|_{\infty}\right)$ such that

$$
|\nabla u(x, \tau)|^{2} \leq C u^{1-\beta}\left(\tau^{-1}+1\right),
$$

for a.e $(x, \tau) \in \Omega \times(0, T)$.

Besides, if $\nabla\left(u_{0}^{\frac{1}{\gamma}}\right) \in L^{\infty}(\Omega)$, with $\gamma=$ $\frac{2}{1+\beta}$, then there is a positive constant $C=$ $C\left(\left\|u_{0}\right\|_{\infty},\left\|\nabla\left(u_{0}^{\frac{1}{\gamma}}\right)\right\|_{\infty}\right)$ such that

$$
|\nabla u(x, \tau)|^{2} \leq C u^{1-\beta}(x, \tau),
$$

for a.e $(x, \tau) \in \Omega \times(0, T)$.

Remark 1. The result of Theorem 1 implies that $u$ is continuous up to the boundary. Furthermore, $u$ is continuous up to $t=0$ if provided $\nabla\left(u_{0}^{\frac{1}{\gamma}}\right) \in L^{\infty}(\Omega)$.

Our next purpose is to study the global existence of solutions. Particularly, we are interested in the complete quenching phenomenon that any solution vanishes identically after a finite time under some circumstances. To state our global existence result and the complete quenching phenomenon, we consider Eq. (1) with source term $\lambda u^{p}$, for any $\lambda>0$. In some of our considerations, a crucial role is played by the first eigenvalue $\lambda_{1}$ of the Dirichlet problem:

$$
\left\{\begin{array}{l}
-\Delta \Phi=\lambda_{1} \Phi \quad \text { in } \Omega \\
\Phi(x)=0, \quad \text { on } \partial \Omega
\end{array}\right.
$$

where $\Phi$ is the first normalized eigenfunction, $\int_{\Omega} \Phi(x) d x=1$. It is known that $\lambda_{1}$ decreases when the measure of the spatial domain $\Omega$ increases. Then, we have a result of global existence of solution.

(2) Theorem 2. Let $u_{0} \in L^{\infty}(\Omega)$, and $f(u)=\lambda u^{p}$, for $\lambda>0$. Assume that there are an open 
bounded domain $\Omega_{0}$, and a positive real number $k_{0}$ such that $\Omega \subset \subset \Omega_{0}$, and

$$
\left\{\begin{array}{r}
u_{0}(x) \leq k_{0} \Phi_{\Omega_{0}}(x), \quad \text { for a.e } x \in \Omega, \\
k_{0} \lambda_{1, \Omega_{0}} \Phi_{\Omega_{0}}(x)+k_{0}^{-\beta} \Phi_{\Omega_{0}}^{-\beta}(x) \geq \lambda\left(k_{0} \Phi_{\Omega_{0}}(x)\right)^{p} \\
\forall(x, t) \in \Omega \times(0, \infty),
\end{array}\right.
$$

where $\lambda_{1, \Omega_{0}}$ and $\Phi_{\Omega_{0}}$ are the first eigenvalue and the first eigenfunction of problem Eq. (5) corresponding to $\Omega_{0}$. Then, any solution $v$ of $E q$. (1) exists globally and satisfies

$$
v(x, t) \leq k_{0} \Phi_{\Omega_{0}}(x), \quad \text { in } \Omega \times(0, \infty) .
$$

Remark 2. For a given $\lambda>0$; if $u_{0}$ is small enough then Eq. (6) holds, and conversely. Thus, we obtain the global existence of solutions if provided either $u_{0}$ or $\lambda$ is small enough.

Next, we give the complete quenching results.

Theorem 3. Let $u_{0} \in L^{\infty}(\Omega)$, and $f(u)=\lambda u^{p}$. For a given $\lambda>0$, then every solution of $E q$. (1) is extinct after a finite time if provided that $\left\|u_{0}\right\|_{\infty}$ is small enough.

Theorem 4. For a given $u_{0} \in L^{\infty}(\Omega)$, there is a real number $\lambda_{0}>0$ such that every solution of $E q$. (1) quenches after a finite time if provided $\lambda \in\left(0, \lambda_{0}\right)$.

Finally, we study the global nonexistence of solutions of Eq. (1), the so called finite time blow-up, see [15], [16], [12], [11], [25], [26], [30]. In this paper, we point out some criteria on initial data $u_{0}$ in order to guarantee the blow-up of solution in a finite time. Thus, it is convenient to introduce the energy functional

$$
\begin{array}{r}
E(t)=\int_{\Omega}\left(\frac{1}{2}|\nabla u(t)|^{2}+\frac{1}{1-\beta} u^{1-\beta}(t)\right. \\
\left.-\frac{1}{p+1} u^{p+1}(t)\right) d x
\end{array}
$$

Then we have a blowing up result as follows:

Theorem 5. Let $u_{0} \in L^{\infty}(\Omega) \cap H_{0}^{1}(\Omega)$. Suppose that $f(u)=u^{p}$, for $p>1$, and $E(0) \leq 0$. Let $u$ be a solution of Eq. (1). Then, $u$ blows up in a finite time.
The paper is organized as follows: In the next section, we prove some gradient estimates for the approximating solutions. In Sec. 3 . we shall prove the local existence results. The global existence of solutions and the complete quenching phenomenon will be considered in Sec. 4. Section 5. is devoted to study the non-global existence of solution. In the final Section, we give some simulation in order to illustrate the interesting phenomenon: quenching versus blow-up.

Several notations which will be used through this paper are the following: we denote by $C$ a general positive constant, possibly varying from line to line. Furthermore, the constants which depend on parameters will be emphasized by using parentheses. For example, $C=C(p, \beta, \tau)$ means that $C$ depends on $p, \beta, \tau$. And $S u p p(f)$ is denoted as the support compact of $f$.

\section{Gradient estimate for the approximate solutions}

In this section, we consider the regularized problem $\left(P_{\varepsilon, \eta}\right)$ of Eq. (1) as follows:

$$
\left\{\begin{array}{lr}
\partial_{t} u-\Delta u+g_{\varepsilon}(u)=u^{p} & \text { in } \Omega \times(0, \infty), \\
u=\eta & \text { on } \partial \Omega \times(0, \infty), \\
u(0)=u_{0}+\eta & \text { on } \Omega
\end{array}\right.
$$

for any $0<\eta<\varepsilon$, with $g_{\varepsilon}(s)=\psi_{\varepsilon}(s) s^{-\beta}$, $\psi_{\varepsilon}(s)=\psi\left(\frac{s}{\varepsilon}\right)$, and $\psi \in \mathcal{C}^{\infty}(\mathbb{R})$ is a nondecreasing function on $\mathbb{R}$ such that $\psi(s)=0$ for $s \leq 1$, and $\psi(s)=1$ for $s \geq 2$. Note that $g_{\varepsilon}$ is a globally Lipschitz function for any $\varepsilon>0$. We will show that solution $u_{\varepsilon, \eta}$ of equation $\left(P_{\varepsilon, \eta}\right)$ tends to a solution of Eq. (1) as $\eta, \varepsilon \rightarrow 0$. In passing to the limit, we need to derive some gradient estimates for solution $u_{\varepsilon, \eta}$. Then, we have the following result:

Lemma 1. Let $u_{0} \in \mathcal{C}_{c}^{\infty}(\Omega), u_{0} \neq 0$. There exists a classical unique solution $u_{\varepsilon, \eta}$ of $\left(P_{\varepsilon, \eta}\right)$ in $\Omega \times(0, T)$.

i) Moreover, there is a constant $C>0$ only depending on $\beta, T, f,\left\|u_{0}\right\|_{\infty}$ such that

$$
\left|\nabla u_{\varepsilon, \eta}(x, \tau)\right|^{2} \leq C u_{\varepsilon, \eta}^{1-\beta}(x, \tau)\left(\tau^{-1}+1\right),
$$


for any $(x, \tau) \in \Omega \times(0, T)$.

ii) If $\nabla\left(u_{0}^{\frac{1}{\gamma}}\right) \in L^{\infty}(\Omega)$, then we get

$$
\left|\nabla u_{\varepsilon, \eta}(x, \tau)\right|^{2} \leq C u_{\varepsilon, \eta}^{1-\beta}(x, \tau),
$$

for any $(x, \tau) \in \Omega \times(0, T)$, with $C>0$ merely depends on $\beta, T,\left\|u_{0}\right\|_{\infty},\left\|\nabla\left(u_{0}^{\frac{1}{\gamma}}\right)\right\|_{\infty}$.

Proof. The proof of this lemma is similar to the one in [20]. Thus, we skip it and refer to Lemma $3.1,[20$.

Thanks to Lemma 1 and the classical regularity (see [14]), we can pass to the limit as $\eta \rightarrow 0$ in problem $\left(P_{\varepsilon, \eta}\right)$. Then, problem $\left(P_{\varepsilon}\right)$ :

$$
\left\{\begin{array}{lr}
\partial_{t} u-\Delta u+g_{\varepsilon}(u)=u^{p} & \text { in } \Omega \times(0, \infty), \\
u=0 & \text { on } \partial \Omega \times(0, \infty), \\
u(0)=u_{0} & \text { on } \Omega
\end{array}\right.
$$

has a unique solution $u_{\varepsilon}$. Note that $u_{\varepsilon}$ also satisfies Lemma 1.

\section{Local existence}

In this section, we consider a local existence of solution of problem Eq. (1). We give the proof of Theorem 1 .

Proof of Theorem 1. Let $u_{\varepsilon}$ be a unique solution of problem $\left(P_{\varepsilon}\right)$ in $\Omega \times(0, T)$. Then, we show that $\left\{u_{\varepsilon}\right\}_{\varepsilon>0}$ is a non-decreasing sequence. Indeed, we have

$$
g_{\varepsilon_{1}}(s) \geq g_{\varepsilon_{2}}(s), \text { for any } 0<\varepsilon_{1}<\varepsilon_{2} .
$$

This implies that $u_{\varepsilon_{1}}$ is a sub-solution of the equation satisfied by $u_{\varepsilon_{2}}$. Therefore, the comparison principle yields

$$
u_{\varepsilon_{1}} \leq u_{\varepsilon_{2}}, \quad \text { in } \Omega \times(0, T), \quad \forall \varepsilon_{1}<\varepsilon_{2},
$$

so the conclusion follows. Consequently, there is a nonnegative function $u$ such that $u_{\varepsilon} \downarrow u$ as $\varepsilon \rightarrow 0^{+}$.

Obviously, we have from the comparison principle

$$
u_{\varepsilon} \leq \Gamma(t)=\left(\left\|u_{0}\right\|_{\infty}^{1-p}-(p-1) t\right)^{1-p},
$$

for any $(x, t) \in \Omega \times\left(0, T_{0}\right), T_{0}=\frac{\left\|u_{0}\right\|_{\infty}^{1-p}}{p-1}$.

Integrating equation $\left(P_{\varepsilon}\right)$ on $\Omega \times(0, T)$ yields

$$
\begin{gathered}
\int_{\Omega} u_{\varepsilon}(x, T) d x-\int_{0}^{T} \int_{\partial \Omega} \nabla u_{\varepsilon} \cdot \mathbf{n} d \sigma d s \\
+\int_{0}^{T} \int_{\Omega} g_{\varepsilon}\left(u_{\varepsilon}\right) d x d s=\int_{\Omega} u_{\varepsilon}(x, 0) d x \\
\quad+\int_{0}^{T} \int_{\Omega} u_{\varepsilon}^{p} d x d s, \forall T \in\left(0, T_{0}\right),
\end{gathered}
$$

where $\mathbf{n}$ is the unit outward normal vector of $\partial \Omega$.

Since $\nabla u_{\varepsilon} \cdot \mathbf{n} \leq 0$, we obtain

$$
\begin{aligned}
\int_{0}^{T} \int_{\Omega} g_{\varepsilon}\left(u_{\varepsilon}\right) d x d s \leq & \int_{\Omega}\left(u_{0}(x)+\varepsilon\right) d x \\
& +\int_{0}^{T} \int_{\Omega} u_{\varepsilon}^{p} d x d s
\end{aligned}
$$

This inequality and the boundedness of $u_{\varepsilon}$ above imply that $\left\|g_{\varepsilon}\left(u_{\varepsilon}\right)\right\|_{L^{1}(\Omega \times(0, T))}$ is bounded by a constant not depending on $\varepsilon$.

Thanks to Fatou's lemma, there is a function $\Upsilon \in L^{1}(\Omega \times(0, T))$ such that

$$
\liminf _{\varepsilon \rightarrow 0} g_{\varepsilon}\left(u_{\varepsilon}\right)=\Upsilon, \quad \text { in } L^{1}(\Omega \times(0, T)) .
$$

Next, the monotonicity of $\left\{u_{\varepsilon}\right\}_{\varepsilon>0}$ deduces

$$
g_{\varepsilon}\left(u_{\varepsilon}\right)(x, t) \geq g_{\varepsilon}\left(u_{\varepsilon}\right) \chi_{\{u>0\}}(x, t),
$$

for $(x, t) \in \Omega \times(0, T)$, so

$\liminf _{\varepsilon \rightarrow 0} g_{\varepsilon}\left(u_{\varepsilon}\right)(x, t)=\Upsilon(x, t) \geq u^{-\beta} \chi_{\{u>0\}}(x, t)$,

for $(x, t) \in \Omega \times(0, T)$. Then, $u^{-\beta} \chi_{\{u>0\}}$ is integrable on $\Omega \times(0, T)$.

Actually, we shall prove

$$
\Upsilon=u^{-\beta} \chi_{\{u>0\}}, \quad \text { in } L^{1}(\Omega \times(0, T)) .
$$

On the other hand, we use a result of gradient convergence of Boccardo et al., [3], 2] in order to obtain

$$
\nabla u_{\varepsilon} \stackrel{\varepsilon \rightarrow 0}{\longrightarrow} \nabla u, \quad \text { for a.e }(x, t) \in \Omega \times(0, T) .
$$

As a result, $\nabla u$ fulfills estimate Eq. (3) for a.e $(x, t) \in \Omega \times(0, T)$, and for any $\tau>0$

$\nabla u_{\varepsilon} \stackrel{\varepsilon \rightarrow 0}{\longrightarrow} \nabla u, \quad$ in $L^{r}(\Omega \times(\tau, T)), \quad \forall r \in[1, \infty)$. 
Now, it suffices to demonstrate that $u$ satisfies Eq. (1) in the sense of distribution.

For any $\eta>0$ fixed, we use the test function $\psi_{\eta}\left(u_{\varepsilon}\right) \phi$, for any $\phi \in \mathcal{C}_{c}^{\infty}(\Omega \times(0, T))$, to the equation satisfied by $u_{\varepsilon}$. Then, using integration by parts yields

$$
\begin{array}{r}
\int_{\text {Supp }(\phi)}\left(-\Psi_{\eta}\left(u_{\varepsilon}\right) \phi_{t}+\frac{1}{\eta}\left|\nabla u_{\varepsilon}\right|^{2} \psi^{\prime}\left(\frac{u_{\varepsilon}}{\eta}\right) \phi\right. \\
+\nabla u . \nabla \phi \psi_{\eta}\left(u_{\varepsilon}\right)+g_{\varepsilon}\left(u_{\varepsilon}\right) \psi_{\eta}\left(u_{\varepsilon}\right) \phi \\
\left.+u_{\varepsilon}^{p} \psi_{\eta}\left(u_{\varepsilon}\right) \phi\right) d x d s=0
\end{array}
$$

with $\Psi_{\eta}(u)=\int_{0}^{u} \psi_{\eta}(s) d s$. Note that $\psi_{\eta}($.$) plays$ a role in avoiding the singularity of the term $u^{-\beta} \chi_{\{u>0\}}$ as $u$ is near 0 . Thus, there is no problem of going to the limit as $\varepsilon \rightarrow 0$ in the indicated equation in order to obtain

$$
\begin{array}{r}
\int_{S u p p(\phi)}\left(-\Psi_{\eta}(u) \phi_{t}+\frac{1}{\eta}|\nabla u|^{2} \psi^{\prime}\left(\frac{u}{\eta}\right) \phi\right. \\
+\nabla u \cdot \nabla \phi \psi_{\eta}(u)+u^{-\beta} \psi_{\eta}(u) \phi \\
\left.+f(u, x, s) \psi_{\eta}(u) \phi\right) d x d s=0 .
\end{array}
$$

Next, we go to the limit as $\eta \rightarrow 0$ in the last equation.

By Eq. (14), Eq. 15), and the integration of $u^{-\beta} \chi_{\{u>0\}}$ in $\Omega \times(0, T)$, it is not difficult to verify

$$
\left\{\begin{array}{l}
\lim _{\eta \rightarrow 0} \int_{\operatorname{Supp}(\phi)} \Psi_{\eta}(u) \phi_{t} d x d s=\int_{\text {Supp }(\phi)} u \phi_{t} d x d s, \\
\lim _{\eta \rightarrow 0} \int_{\text {Supp }(\phi)} \nabla u \cdot \nabla \phi \psi_{\eta}(u) d x d s=\int_{\text {Supp }(\phi)} \nabla u . \nabla \phi d x d s, \\
\lim _{\eta \rightarrow 0} \int_{\operatorname{Supp}(\phi)} u^{-\beta} \psi_{\eta}(u) \phi d x d s=\int_{\operatorname{Supp}(\phi)} u^{-\beta} \chi_{\{u>0\}} \phi, \\
\lim _{\eta \rightarrow 0} \int_{\operatorname{Supp}(\phi)} u^{p} \psi_{\eta}(u) \phi d x d s=\int_{\operatorname{Supp}(\phi)} u^{p} \phi d x d s .
\end{array}\right.
$$

Next, we show that

$$
\lim _{\eta \rightarrow 0} \int_{S u p p(\phi)} \frac{1}{\eta}|\nabla u|^{2} \psi^{\prime}\left(\frac{u}{\eta}\right) \phi d x d s=0 .
$$

In fact, since $u$ satisfies estimate Eq. (3), we have

$$
\begin{aligned}
& \frac{1}{\eta} \int_{\text {Supp }(\phi)}|\nabla u|^{2}\left|\psi^{\prime}\left(\frac{u}{\eta}\right) \phi\right| d x d s \\
\leq & C \frac{1}{\eta} \int_{\operatorname{Supp}(\phi) \cap\{\eta<u<2 \eta\}} u^{1-\beta} d x d s \\
\leq & 2 C \int_{\operatorname{Supp}(\phi) \cap\{\eta<u<2 \eta\}} u^{-\beta} d x d s,
\end{aligned}
$$

where $\operatorname{Supp}(\phi)$ means the support compact of $\phi$, and the constant $C>0$ is independent of $\eta$. Since $u^{-\beta} \chi_{\{u>0\}}$ is integrable on $\Omega \times(0, T)$, we obtain

$$
\lim _{\eta \rightarrow 0} \int_{\operatorname{Supp}(\phi) \cap\{\eta<u<2 \eta\}} u^{-\beta} d x d s=0,
$$

which implies the conclusion Eq. (17). A combination of Eq. (16) and Eq. (17) deduces

$$
\begin{array}{r}
\int_{S u p p(\phi)}\left(-u \phi_{t}+\nabla u . \nabla \phi\right. \\
\left.+u^{-\beta} \chi_{\{u>0\}} \phi+f(u, x, s) \phi\right) d x d s=0 .
\end{array}
$$

In other words, $u$ satisfies Eq. (1) in $\mathcal{D}^{\prime}(\Omega \times$ $(0, T))$.

As mentioned above, we prove Eq. (13) now. From equation $\left(P_{\varepsilon}\right)$, we have

$$
\begin{array}{r}
\int_{\operatorname{Supp}(\phi)}\left(-u_{\varepsilon} \phi_{t}+\nabla u_{\varepsilon} \cdot \nabla \phi\right. \\
\left.+g_{\varepsilon}\left(u_{\varepsilon}\right) \phi+f\left(u_{\varepsilon}, x, s\right) \phi\right) d x d s=0,
\end{array}
$$

for any $\phi \in \mathcal{C}_{c}^{\infty}(\Omega \times(0, T)), \phi \geq 0$.

Then, letting $\varepsilon \rightarrow 0$ deduces

$$
\begin{array}{r}
\int_{\text {Supp }(\phi)}\left(-u \phi_{t}+\nabla u . \nabla \phi\right) d x d s+ \\
\lim _{\varepsilon \rightarrow 0} \int_{\operatorname{Supp}(\phi)} g_{\varepsilon}\left(u_{\varepsilon}\right) \phi d x d s \\
+\int_{\text {Supp }(\phi)} f(u, x, t) \phi d x d s=0 .
\end{array}
$$

By Eq. 18) and Eq. 19, we get

$$
\begin{aligned}
& \lim _{\varepsilon \rightarrow 0} \int_{\operatorname{Supp}(\phi)} g_{\varepsilon}\left(u_{\varepsilon}\right) \phi d x d s \\
= & \int_{\operatorname{Supp}(\phi)} u^{-\beta} \chi_{\{u>0\}} \phi d x d s .
\end{aligned}
$$


According to Eq. (11), Eq. (20) and Fatou's lemma, we obtain

$$
\begin{aligned}
& \int_{\operatorname{Supp}(\phi)} u^{-\beta} \chi_{\{u>0\}} \phi d x d s \geq \int_{\operatorname{Supp}(\phi)} \Upsilon \phi d x d s, \\
& \forall \phi \in \mathcal{C}_{c}^{\infty}(\Omega \times(0, T)), \phi \geq 0 .
\end{aligned}
$$

The last inequality and Eq. (12) yield conclusion Eq. 13.

The conclusion $u \in \mathcal{C}\left([0, T] ; L^{1}(\Omega)\right)$ is well known, so we skip its proof and refer to the compactness result of J. Simon, [33. Thus, $u$ is a weak solution of Eq. (1).

To complete the proof of Theorem 1 it remains to show that $u$ is the maximal solution of Eq. (1).

Let $v$ be any weak solution of Eq. (1) on $\Omega \times$ $(0, T)$. Then, we have

$$
v(x, t) \leq u(x, t), \quad \text { for a.e }(x, t) \in \Omega \times(0, T) .
$$

In fact, we observe that

$$
g_{\varepsilon}(v) \leq v^{-\beta} \chi_{\{v>0\}}, \quad \forall \varepsilon>0 .
$$

Thus,

$\partial_{t} v-\Delta v+g_{\varepsilon}(v) \leq f(v, x, t), \quad$ in $\mathcal{D}^{\prime}(\Omega \times(0, T))$,

which implies that $v$ is a sub-solution of equation $\left(P_{\varepsilon}\right)$.

By the comparison principle, we get

$$
v(x, t) \leq u_{\varepsilon}(x, t), \quad \text { for a.e }(x, t) \in \Omega \times(0, T) .
$$

Letting $\varepsilon \rightarrow 0$ yields the result.

\section{Global existence and complete quenching phenomenon}

In this part, we study the global existence of solution and the complete quenching phenomenon through proving Theorem 2 and Theorem 3. Since $u$ is the maximal solution, then it suffices to work on $u$.
Proof of Theorem 2, Let $u$ be the maximal solution of Eq. (1) in $\Omega \times(0, T)$. To prove that $u$ exists globally, we show that $u$ is bounded by a constant not depending on $t$.

We first remind that $\inf _{x \in \Omega}\left\{\Phi_{\Omega_{0}}(x)\right\}>0$, for $\Omega \subset \subset \Omega_{0}$.

Thus, for any $\varepsilon \in\left(0, \frac{1}{2} \inf _{x \in \Omega}\left\{k_{0} \Phi_{\Omega_{0}}(x)\right\}\right)$, we have $g_{\varepsilon}\left(k_{0} \Phi_{\Omega_{0}}\right)=k_{0}^{-\beta} \Phi_{\Omega_{0}}^{-\beta}$.

Put

$$
\mathcal{L}_{\varepsilon}(v):=v_{t}-\Delta v+g_{\varepsilon}(v)-\lambda v^{p} .
$$

Then,

$\mathcal{L}_{\varepsilon}\left(k_{0} \Phi_{\Omega_{0}}\right)=k_{0} \lambda_{1}\left(\Omega_{0}\right) \Phi_{\Omega_{0}}+k_{0}^{-\beta} \Phi_{\Omega_{0}}^{-\beta}-\lambda\left(k_{0} \Phi_{\Omega_{0}}\right)^{p}$.

By Eq. (6), we observe that $\mathcal{L}_{\varepsilon}\left(k_{0} \Phi_{\Omega_{0}}\right) \geq 0$, in $\Omega \times(0, T)$. Therefore, $k_{0} \Phi_{\Omega_{0}}$ is a super-solution of equation $\left(P_{\varepsilon}\right)$. The strong comparison theorem yields

$$
u_{\varepsilon}(x, t) \leq k_{0} \Phi_{\Omega_{0}}(x), \quad \forall(x, t) \in \Omega \times(0, T) .
$$

Since $u_{\varepsilon}$ is bounded on $\Omega \times(0, T)$, the standard argument allows us to extend the existence of $u_{\varepsilon}$ on $\Omega \times\left(0, T+\delta_{0}\right), \Omega \times\left(0, T+2 \delta_{0}\right), \ldots$, for some $\delta_{0}>0$, thereby proves the global existence of solution $u_{\varepsilon}$. By the monotonicity of $u_{\varepsilon}, u$ must exist globally, and the conclusion Eq. (7) follows immediately.

Next, we will show that for a given $\lambda>0$, the maximal solution $u$ must vanish identically after a finite time if $\left\|u_{0}\right\|_{\infty}$ is small enough.

Proof of Theorem 3 Since $\left\|u_{0}\right\|_{\infty}$ is small enough, we can choose a real number $k_{0}>0$ small as well, and an open bounded domain $\Omega_{0}$ containing $\Omega$, such that Eq. (6) holds. Thanks to Theorem 2, the maximal solution $u$ exists globally, and it is bounded by $M=k_{0} \sup _{x \in \Omega}\left\{\Phi_{\Omega_{0}}(x)\right\}$.

Using the test function $u$ to Eq. (1) gives us

$$
\begin{array}{r}
\frac{1}{2} \frac{d}{d t} \int_{\Omega} u^{2}(t) d x+\int_{\Omega}\left(|\nabla u(t)|^{2}+u^{1-\beta}(t)\right) d x= \\
\lambda \int_{\Omega} u^{p+1} d x, \quad \forall t>0 .
\end{array}
$$


Since $u \leq M$, we have

$$
\begin{array}{r}
\frac{1}{2} \frac{d}{d t} \int_{\Omega} u^{2}(t) d x+\int_{\Omega}\left(|\nabla u(t)|^{2}+u^{1-\beta}(t)\right) d x \\
\leq \lambda M^{p+\beta} \int_{\Omega} u^{1-\beta}(t) d x, \quad \forall t>0 .
\end{array}
$$

Or

$$
\begin{array}{r}
\frac{1}{2} \frac{d}{d t} \int_{\Omega} u^{2}(t) d x+\int_{\Omega}|\nabla u(t)|^{2} d x+ \\
\left(1-c_{M}\right) \int_{\Omega} u^{1-\beta}(t) d x \leq 0,
\end{array}
$$

where $c_{M}=\lambda M^{p+\beta}$. Note that $M$ is as small as $\left\|u_{0}\right\|_{\infty}$. Thus, if $\left\|u_{0}\right\|_{\infty}$ is small enough then we get $\left(1-c_{M}\right)>c_{0}>0$.

It follows from Eq. 22 that

$\frac{1}{2} \frac{d}{d t} \int_{\Omega} u^{2}(t) d x+c_{0} \int_{\Omega}\left(|\nabla u(t)|^{2} d x+u^{1-\beta}(t)\right) d x \leq 0$.

Now, using Garliardo-Nirenberg's inequality [31] yields

$$
\begin{aligned}
& \|u(t)\|_{L^{2}(\Omega)} \leq c\|\nabla u(t)\|_{L^{2}(\Omega)}^{\theta}\|u(t)\|_{L^{1}(\Omega)}^{1-\theta} \\
& =c\left(\int_{\Omega}|\nabla u(t)|^{2} d x\right)^{\frac{\theta}{2}}\left(\int_{\Omega} u(t) d x\right)^{1-\theta},
\end{aligned}
$$

with $\theta=\frac{N+1}{N+2}$. Thus,

$$
\begin{gathered}
\|u(t)\|_{L^{2}(\Omega)} \leq c\left(\int_{\Omega}\left(|\nabla u(t)|^{2}+u(t)\right) d x\right)^{\frac{\theta}{2}+1-\theta} \\
\leq c\left(\int_{\Omega}\left(|\nabla u(t)|^{2}+M^{\beta} u^{1-\beta}(t)\right) d x\right)^{1-\theta / 2} \\
\leq c_{1}\left(\int_{\Omega}\left(|\nabla u(t)|^{2}+u^{1-\beta}(t)\right) d x\right)^{1-\theta / 2},
\end{gathered}
$$

with $c_{1}=c_{1}(\beta, M)>0$.

Then, we obtain

$$
\left(\int_{\Omega} u^{2}(t) d x\right)^{\frac{N+2}{N+3}} \leq c_{2} \int_{\Omega}\left(|\nabla u(t)|^{2}+u^{1-\beta}(t)\right) d x
$$

with $c_{2}=c_{2}(\beta, M, N)>0$.

By Eq. 23) and Eq. (24), there is a positive constant $c_{3}=c_{3}(\beta, M)>0$ such that

$$
y^{\prime}(t)+c_{3} y^{\frac{N+2}{N+3}}(t) \leq 0, \quad \text { for } t>0,
$$

with $y(t)=\int_{\Omega}|u(x, t)|^{2} d x$.

If we can show that there exists a time $t_{0} \in$ $[0, \infty)$ such that $y\left(t_{0}\right)=0$, it follows then from Eq. (25) that $y(t)=0$, for any $t \geq t_{0}$, thereby proves Theorem 3

Indeed, we assume a contradiction that $y(t)>0$, for any $t>0$. Solving the ordinary differential inequality Eq. 25 yields

$y^{\frac{1}{N+3}}(t)+c_{4} t \leq y^{\frac{1}{N+3}}(0)=\left\|u_{0}\right\|_{L^{2}(\Omega)}^{\frac{2}{N+3}}, \quad \forall t>0$,

with $c_{4}=\frac{c_{3}}{N+3}$. This leads to a contradiction as $t$ is sufficiently large. Thus, $u$ must quench after a finite time.

Similarly, for a given initial data $u_{0}$, we also obtain the complete quenching result for the case $\lambda$ small. Thus, Theorem 4 follows.

Remark 3. Inequality Eq. (26) implies that the extinction time of $u$, denoted by $T^{\star} \leq$ $\frac{\left\|u_{0}\right\|_{L^{2}(\Omega)}^{\frac{2}{N+2}}}{c_{4}}$.

\section{Non-global existence of solutions}

In this section, we study the non-global existence of solutions of Eq. (1). We give the proof of Theorem 5 .

Proof of Theorem 5. By multiplying $u$ (resp. $u_{t}$ ) to Eq. (1), we have the following integral equations:

$$
\begin{array}{r}
\frac{1}{2} \frac{d}{d t} \int_{\Omega} u^{2}(x, t) d x=-\int_{\Omega}\left(|\nabla u(x, t)|^{2}+\right. \\
\left.u^{1-\beta}(x, t)-u^{q+1}(x, t)\right) d x,
\end{array}
$$

and

$$
\begin{array}{r}
\int_{0}^{t} \int_{\Omega}\left|u_{t}\right|^{2} d x d s+\int_{\Omega}\left(\frac{1}{2}|\nabla u(t)|^{2}+\frac{1}{1-\beta} u^{1-\beta}(t)\right. \\
\left.-\frac{1}{q+1} u^{q+1}(t)\right) d x= \\
\int_{\Omega}\left(\frac{1}{2}\left|\nabla u_{0}\right|^{2}+\frac{1}{1-\beta} u_{0}^{1-\beta}-\frac{1}{q+1} u_{0}^{q+1}\right) d x,
\end{array}
$$


see [30, 32]. By combining Eq. 27 and Eq. 28, we obtain

$$
\begin{array}{r}
\frac{1}{2} \frac{d}{d t} \int_{\Omega} u^{2}(x, t) d x=-2 E(t) \\
+\frac{1+\beta}{1-\beta} \int_{\Omega} u^{1-\beta}(x, t) d x+\frac{q-1}{q+1} \int_{\Omega} u^{q+1}(x, t) d x .
\end{array}
$$

Since $E(0) \leq 0$, Eq. 28 implies $E(t) \leq 0$, for any $t>0$. It follows then from the last inequality that

$$
\frac{1}{2} \frac{d}{d t} \int_{\Omega} u^{2}(x, t) d x \geq \frac{q-1}{q+1} \int_{\Omega} u^{q+1} d x .
$$

By Holder's inequality, we get

$$
\int_{\Omega} u^{2} d x \leq\left(\int_{\Omega} u^{q+1} d x\right)^{\frac{2}{q+1}}|\Omega|^{\frac{q-1}{q+1}}
$$

From Eq. 29 and Eq. (30), we obtain

$$
y^{\prime}(t) \geq C y^{\frac{q+1}{2}}(t)
$$

with $y(t)=\int_{\Omega} u^{2}(x, t) d x$, and $C=\frac{2(q-1)}{(q+1)|\Omega|^{\frac{q-1}{2}}}$.

This inequality implies that $y(t) \rightarrow+\infty$ as $t \rightarrow T_{0}^{-}$, with $T_{0}=\frac{4\left\|u_{0}\right\|_{L^{2}(\Omega)}^{1-q}}{(q+1)|\Omega|^{\frac{q-1}{2}}}$.

\section{Simulation results}

In this part, we will illustrate our theoretical results with some numerical experiences. In the sequel, we consider Eq. (1) with $p=2, \beta=$ $0.8, I=(0, L)$, and $u_{0}(x)=x(1-x / L)$. Our numerical scheme mimics the one in the paper of [10. Similarly, we use the linear finite elements with mass lumping in a uniform mess for the space variable to discretize our Eq. (1). The reader who is interested in detail can find in [10].

We fix $L=2$.

With $\lambda=14$, the maximal solution of Eq. (1) vanished after $t=0.36$, see Fig. 1. While $\lambda=$ 15, the maximal solution of Eq. (1) blows up at $t=0.33$, see Fig. 2.

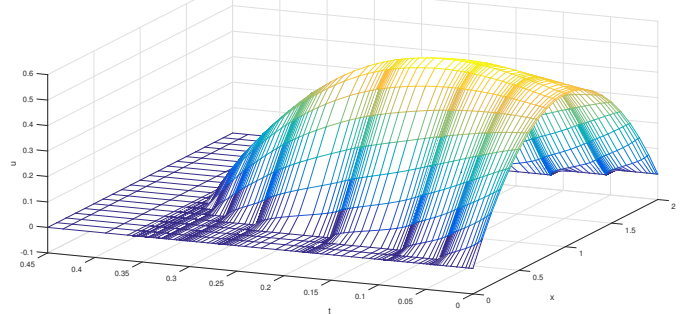

Fig. 1: Evolution of the maximal solution of Eq. 11.

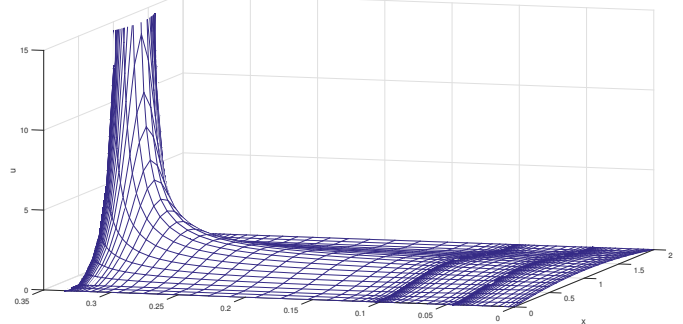

Fig. 2: Evolution of the maximal solution of Eq. 11.

\section{References}

[1] BANKS, H. T. Modeling and control in the biomedical sciences. Heidelberg: Springer Berlin Heidelberg, 1975.

[2] BOCCARDO, L. and T. GALLOUET. Nonlinear elliptic and parabolic equations involving measure data. Journal of Functional Analysis. 1989, vol. 87, iss. 1, pp. $149-169$.

[3] BOCCARDO, L. and F. MURAT. Almost everywhere convergence of the gradients of solutions to Elliptic and Parabolic equations. Nonlinear Analysis: Theory, Methods \& Applications. 1992, vol. 19, iss. 6, pp. 581-597.

[4] CODDINGTON, E. and LEVINSON N. Theory of Ordinary Differential Equations. New York: McGraw-Hill, 1955.

[5] DAO, A. N., J. I. DIAZ and P. SAUVY. Quenching phenomenon of singular parabolic problems with $L^{1}$ initial data. 
Electronic Journal of Differential Equations. 2016, vol. 2016, no. 136, pp. 1-16.

[6] DAO, A. N., J. I. DIAZ and H. V. KHA. Complete quenching phenomenon and instantaneous shrinking of compact support of degenerate parabolic equations with nonlinear absorption. To appear in Proceedings of the Royal Society of Edinburgh, Section A: Mathematics. 2017.

[7] DAO, A. N. and J. I. DIAZ. The extinction versus the blow-up: Global and nonglobal existence of solutions of source types of degenerate parabolic equations with a singular absorption. Journal of Differential Equations. 2017, vol. 263, iss. 10, pp. 67646804.

[8] DAO, A. N. and J. I. DIAZ. A gradient estimate to a degenerate parabolic equation with a singular absorption term: global and local quenching phenomena. Journal of Mathematical Analysis and Applications. 2016, vol. 437, iss. 1, pp. 445473. ISSN 0022-247x.

[9] DAVILA, J. and M. MONTENEGRO. Existence and asymptotic behavior for a singular parabolic equation. Transactions of the American Mathematical Society. 2004, vol. 357 , iss. 5, pp. 1801-1828.

[10] FERREIRA, R. and A. D. PABLO. Numerical Blow-up for the p-Aplacian Equation with a Source. Computational Methods in Applied Mathematics. 2005, vol. 5, iss. 2, pp. 137-154.

[11] GALAKTIONOV, V. A. Blow-up for quasilinear heat equations with critical Fujita's exponents. Proceedings of the Royal Society of Edinburgh: Section A Mathematics. 1994, vol. 124, iss. 3, pp. 517-525.

[12] GALAKTIONOV, V. and J. L. VAZQUEZ. The problem Of blow-up in nonlinear parabolic equations. Discrete and Continuous Dynamical Systems. 2002, vol. 8, iss. 2, pp. 399-525.

[13] KAWOHL B. Remarks on Quenching. Doc. Math., J. DMV. 1996, vol. 1, pp. 199-208.
[14] LADYZENSKAJA O. A., V. A. SOLONNIKOV and N. N. URALCEVA. Linear and Quasi-Linear Equations of Parabolic Type. American Mathematical Soc., 1988.

[15] LEVINE H. A. The role of critical exponents in blowup theorems. SIAM Review. 1990, vol. 32, iss. 2, pp. 262-288.

[16] LEVINE H. A. Quenching and beyond: a survey of recent results. Internat. Ser. Math. Sci. Appl.. 1993, vol. 2, pp. 501-512.

[17] MONTENEGRO, M. Complete quenching for singular parabolic problems. Journal of Mathematical Analysis and Applications. 2011, vol. 384, iss. 2, pp. 591-596.

[18] PHILLIPS D. Existence of solutions of quenching problems. Applicable Analysis. 1987, vol. 24, iss. 4 , pp. 253-264.

[19] STRIEDER W. and R. ARIS. Variational Methods Applied to Problems of Diffusion and Reaction. Berlin: Springer-Verlag, 1973.

[20] WINKLER M. Nonuniqueness in the quenching problem. Mathematische Annalen. 2007, vol. 339, iss. 3, pp. 559-597.

[21] BELAUD Y. and J. I. DIAZ. Abstract results on the finite extinction time property: application to a singular parabolic equation. Journal of Convex Analysis. 2010, vol. 17 , pp. $827-860$.

[22] DIAZ J. I. Nonlinear partial differential equations and free boundaries, Research Notes in Mathematics. Boston: Pitman Advanced Pub, 1985.

[23] DIAZ J. I. On the free boundary for quenching type parabolic problems via local energy methods. Communications on Pure and Applied Analysis. 2014, vol. 13, iss. 5, pp. 1799-1814.

[24] EVANS L. C. and R. GARIEPY. Measure theory and fine properties of functions. Boca Raton: CRC Press, 1992.

[25] GALAKTIONOV, V. and J. L. VAZQUEZ. Necessary and sufficient conditions of complete blow up and extinction for onedimensional quasilinear heat equations. 
Archive for Rational Mechanics and Analysis. 1995, vol. 129, iss. 3, pp. 225-244.

[26] GALAKTIONOV, V. and J. L. VAZQUEZ. Continuation of blowup solutions of nonlinear heat equations in several space dimensions. Communications on Pure and Applied Mathematics. 1997, vol. 50, iss. 1, pp. 1-67.

[27] KAPLAN, S. On the growth of solutions of quasilinear parabolic equations. Communications on Pure and Applied Mathematics. 1963, vol. 16, iss. 3, pp. 305-333.

[28] KAWARADA, H. On solutions of initialboundary problem for $u_{t}=u_{x x}+\frac{1}{1-u}$. Publications of the Research Institute for Mathematical Sciences. 1974, vol. 10, iss. 3, pp. 729-736.

[29] KAWOHL, B. and R. KERSNER. On degenerate diffusion with very strong absorption. Mathematical Methods in the Applied Sciences. 1992, vol. 15, iss. 7, pp. 469-477.

[30] QUITTNER, P. and P. SOUPLET. Superlinear parabolic problems: blowup, global existence and steady states. Basel: Birkhäuser, 2007.
[31] NIRENBERG, L. On elliptic partial differential equations. Annali della Scuola Normale Superiore di Pisa - Classe di Scienze. 1955, vol. 13, no. 2, pp. 116-162.

[32] SAMARSKII, A. A. Blow-up in quasilinear parabolic equations. New York: De Gruyter, 1995.

[33] SIMON, J. Compact sets in the space $L^{p}(0, T ; B)$. Annali di Matematica Pura ed Applicata. 1987, vol. 196, iss. 1, pp. 65-96.

[34] WEISSLER, F. Existence and nonexistence of global solutions of a semi-linear heat equation. Israel Journal of Mathematics. 1981, vol. 38, iss. 1, pp. 29-40.

\section{About Authors}

Nguyen Anh DAO was born in Binh Thuan province, Vietnam. I received a Ph.D. degree in mathematics, in France in 2013. From April 2014 to August 2017, I was a lecturer at Ton Duc Thang University. Since Sept 2017, I holds a position of researcher at Ton Duc Thang University. My research interest is Analysis and Partial Differential Equations.

"This is an Open Access article distributed under the terms of the Creative Commons Attribution License, which permits unrestricted use, distribution, and reproduction in any medium, provided the original work is properly cited (CC BY 4.0)." 